\title{
X-RAY DIFFRACTION AND COMPUTER SIMULATION STUDIES ON THE STRUCTURE OF HOMOPHASE AND HETEROPHASE INTERFACES IN METALS
}

\author{
I. MAJID, C. A. COUNTERMAN, R. NAJAFABADI $\dagger$ and P. D. BRISTOWE \\ Department of Materials Science and Engineering, MIT, U.S.A. \\ $†$ Department of Materials Science and Engineering, University of Michigan, Ann Arbor, U.S.A.
}

\begin{abstract}
We use the Thin Film X-ray Diffraction (TFXD) technique in conjunction with computer simulation methods to obtain detailed information on the structure of complex interfacial systems in metals. Results for three types of interfaces are presented. These are, a $\Sigma 13$ [001] twist homophase boundary in a Au-Cu 10 at.\% alloy, a $\Sigma 26 / 29$ [001] twist Au-Pd heterophase boundary and finally the $\Sigma 4 / 5[001]$ twist $\mathrm{Ag}-\mathrm{Cu}$ heterophase boundary. For the homophase boundary in $\mathrm{Au}-\mathrm{Cu}$ we demonstrate the utility of the X-ray technique in studying the effects of solute segregation on boundary structure. Calculations predict trends which are in agreement with the experimental observations; however the precise magnitudes of the observed effects are not reproduced by the calculations. For the Au-Pd heterophase boundary semi-quantitative agreement is obtained between the experiments and the calculations (performed using EAM potentials and Molecular Statics). Finally, in the $\mathrm{Ag}-\mathrm{Cu} \Sigma 4 / 5$ system, strong X-ray scattering is observed on the BDL, and is correlated with surprisingly large atomic displaccments in this boundary; fairly good agreement is obtained with computer simulated structures. We conclude with speculations on the usefulness of the simple dislocation model in the $\mathrm{Ag}-\mathrm{Cu}$ [001] twist system.
\end{abstract}

Keywords: A. interfaces, A. metals, C. X-ray diffraction, D. crystal structure, D. defects.

\section{INTRODUCTION}

Interfaces, whether between similar or dissimilar materials are ubiquitous. The gross macroscopic properties of any material are strongly influenced by the properties of interfaces between grains of differing chemistry and orientation. Some such properties are diffusivity, conductivity, fracture strength, cohesion ctc. This is true of virtually all materials in the condensed state, metals, ceramics, semiconductors, superconductors, even polymers. Furthermore, for most materials, all of the properties listed above, and many others, depend sensitively on the structure of the interfaces. A detailed knowledge, first of all, of the structure of interfaces (i.e. both atomic arrangement and chemical identity) and the subsequent correlation of this information with different properties is therefore of much importance.

Experimentally, a variety of techniques exist by which the structure of interfaces may be determined. The most important of these are HRTEM [1], X-ray diffraction [2,3], field-ion microscopy [4] and Auger electron spectroscopy [5]. Of these, only the X-ray diffraction technique is capable, in principle, of providing both geometric and chemical information (in the form of site occupation probabilities) for interfaces. We shall therefore focus on the X-ray technique and use it in conjunction with computer simulation methods to obtain detailed information on the structurc of both homophase and heterophase interfaces in metals.

Computer simulation techniques have been used with considerable success in the past in order to obtain detailed atomistic information on the structure of grain boundaries. While the validity of these calculations has been confirmed experimentally, with considerable sensitivity, for some of the simplest interfacial systems [3], their applicability in the study of more complex interfacial systems remains to be established.

Our motivation here, then, is three-fold. First of all, we would like to use X-ray diffraction to study some of these complex interfacial systems and therefore obtain detailed information on the atomic structure of these systems. Secondly, we would like to compare structure factors obtained by X-ray diffraction with structure factors obtained from realistic simulations using, for example Embedded Atom Method (EAM) potentials [6] - the extent to which the calculations and the experiments agree will therefore serve as a strong indication of the validity of these techniques. (In many instances we find that due to the large number of parameters in a unit cell, i.e. atomic positions $x_{j}, y_{j}, z_{j}$ and site occupation probabilities $p_{j}$, it is not possible to invert the experimentally observed diffraction intensities to obtain a 
unique atomic structure of the interface, in these cases we shall proceed by checking for consistency between experiments and calculations.)

Finally, we would like to point out that the use of the Thin Film X-ray Diffraction (TFXD) technique in obtaining the structures of fully equilibrated homophase and heterophase interfaces is a novel feature of this work, and to the best of our knowledge, this is one of the first such studies of a homogenized heterophase boundary. It is therefore hoped that the present work will serve as an anchor point in establishing the TFXD technique as a powerful tool in the study of both homophase and heterophase interfaces in materials.

In this work we focus on three different boundary types, these are: (a) a $22.6^{\circ}[001]$ twist $\Sigma 13$ $\mathrm{Au}-\mathrm{Cu} \sim 10$ at. $\%$ alloy homo-phase boundary as a model system for the study of segregation; (b) a $10.4^{\circ}$, $\Sigma$ 26/29 Au-Pd hetero-phase boundary; and finally (c) the 26.6 [001] twist $\Sigma$ 4/5 interface in the $\mathrm{Ag}-\mathrm{Cu}$ heterophase system. For each of the above systems we conduct both X-ray diffraction and computer simulation experiments that provide valuable insight into the nature of the atomic relations at the interface.

\section{X-RAY DIFFRACTION TECHNIQUE}

The details of the TFXD technique used in the present work follows closely that described in Refs $[3,7]$. We therefore refer the interested reader to those publications for a more complete and thorough description of the technique. Here we simply mention that the X-ray experiments were performed at the NSLS in Brookhaven Laboratories, using beamlines $\mathrm{X} 20 \mathrm{~A}$ and $\mathrm{C}$. Depending on the interfacial system being studied, both $7.9 \mathrm{keV}$ and $10 \mathrm{keV}$ photons were used, by monochromating with double bounce Ge or Si. In particular it was necessary to use photons with energy below the $\mathrm{CuK}_{\alpha}$ edge in order to reduce background levels (due to fluorescence) for the $\mathrm{Cu} / \mathrm{Ag}$ system.

\section{Au-Cu 10 at. $\% \Sigma 13$ BOUNDARY}

While a fair amount of work has been done on the observation of dislocation structures in alloyed boundaries, with one exception [8], virtually nothing is known experimentally about the detailed atomic structure of alloyed homophase boundaries. In particular, the effect of alloying/segregation on the detailed structure of such boundaries is completely unknown. Here we present results showing clearly how the impurity atoms give rise to a significant and measurable perturbation on the equilibrium structure of the pure boundary.

\section{Sample preparation}

$\mathrm{Au}-\mathrm{Cu}$ alloy thin films were manufactured by coevaporating carefully measured amounts of $\mathrm{Au}$ and $\mathrm{Cu}$ from separate $\mathrm{W}$ boats, in a diffusion pumped bell jar system (base pressure $10^{-7}$ torr) onto cleaved [001] $\mathrm{NaCl}$ substrates maintained at a temperature of $290^{\circ} \mathrm{C}$. The samples were then homogenized in the bell jar for several hours at $400^{\circ} \mathrm{C}$, before being cooled back to room temperature. Auger spectra taken from the surfaces of the freshly evaporated alloy films revealed the presence of oxygen peaks, due to oxidation of the $\mathrm{Cu}$ atoms upon exposure to atmosphere. The single crystal films, still on their $\mathrm{NaCl}$ substrates, were then mounted on a specially designed jig, which allowed the two substrates to be misoriented by a well defined angle, to an accuracy of about $0.5^{\circ}$, without the two thin film surfaces being in contact. The jig was then mounted in a tubular hydrogen furnance in which the surface region of the single crystals was reduced by annealing in a pure hydrogen atmosphere at a temperature of $\sim 450^{\circ} \mathrm{C}$ for a period of $2-3 \mathrm{~h}$. After the surfaces were thus reduced, the temperature in the furnace was lowered to $\sim 425^{\circ} \mathrm{C}$ and the two single crystal faces were brought into contact by an external push/pull feed-through mechanism which also allowed the application of a moderate pressure of $\sim 50 \mathrm{psi}$ on the bicrystal sandwich [9]. The sandwiched thin film combination was then annealed at $425^{\circ} \mathrm{C}$ for $\sim 15-24 \mathrm{~h}$. This annealing schedule was generally sufficient to produce a well bonded interface. For a number of samples an additional anneal in a vacuum furnace, at $\sim 400^{\circ} \mathrm{C}$, for a couple of days, was performed in order to ensure complete homogeneity of the bicrystal alloy film. No systematic differences were found between the two sets of samples. In the sample preparation procedure it was deemed necessary to reduce the surfaces first, and then begin the bonding step in order to ensure that the reduction took place uniformly throughout the entire surface and that no remaining oxide would therefore be present at the interface. Such care is especially important in $\mathrm{X}$-ray diffraction experiments where $\sim \mathrm{mm}^{2}$ of well formed grain boundary regions are necessary in order to give rise to measurable diffraction peaks.

\section{Simulations}

Computer simulations were performed on the $\mathrm{Au}-\mathrm{Cu} 10$ at. \% alloy using the EAM interactions, using the free energy minimization technique of Le Sar et al. [10]. This particular technique was chosen since it is computationally very efficient, and has been thoroughly tested in alloy systems such as $\mathbf{A u}-\mathbf{A g}$, $\mathrm{Au}-\mathrm{Pd}, \mathrm{Cu}-\mathrm{Ni}[11]$. The details are thoroughly described in Refs $[10,11]$ and we refer the reader to 
those publications. Here we note that the simulations were performed at a temperature of $400^{\circ} \mathrm{C}$ at constant pressure, with periodic border conditions in all three directions, the basic simulation cell consisted of a $2 \times 2 \Sigma 13$ cell with a total of 2080 atoms. The free energy functional (with variables $x_{j}, y_{j}, z_{j}$ and $p_{j}$ ) was minimized using a conjugate gradient method.

\section{Results}

The reciprocal lattice associated with the $\Sigma 13$ (BDL) [12] unit cell is shown in Fig. 1. The strongest grain boundary reflections lie on the reciprocal O-lattice associated with a bulk lattice reflection, and these are shown schematically as the large solid circles clustered around a lattice reflection. Two aspects of this BDL deserve special attention. First of all we note that the "distance" from the lattice reflection to the corresponding O-reflection is proportional to the inverse of the dislocation spacing $\left(\sim 1 / d_{0}^{\text {pure }}\right)$ at the interface. Furthermore, we note that when $\mathrm{Cu}$ is alloyed with $\mathrm{Au}$ a change in the bulk lattice parameter from the pure Au value $(4.078 \AA$ ) to that of the corresponding alloy will take place. Hence the bulk 200 lattice reflection for the alloy ( $x$ symbols) will extend further away in reciprocal space from the origin relative to the pure $\mathrm{Au} 200$ lattice reflection (filled squares-this is indicated in Fig. 2). Correspondingly, the dislocation spacing will also change (due to the change in lattice parameter) and the reciprocal $\mathrm{O}$-lattice refiection will extend a distance $\left(\sim 1 / d_{0}^{\text {alloy }}\right)$, in reciprocal space, away from the bulk

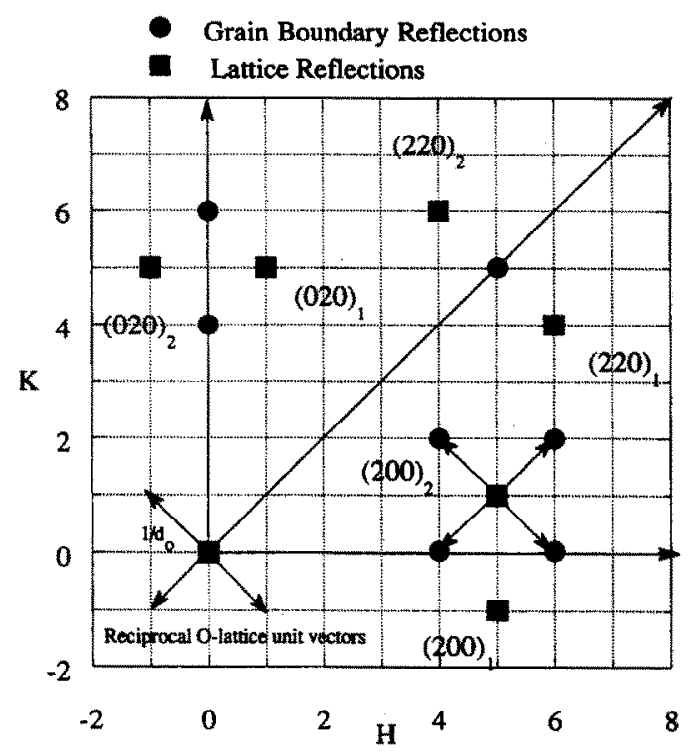

Fig. 1. The boundary diffraction lattice for the $\Sigma 13$ [001] twist boundary is shown. Grain boundary reflections are indicated by solid circles, lattice reflections by solid squares. Reciprocal $O$-lattice unit vectors are indicted by small diagonal arrows emanating from the $(200)_{2}$ reflection.
A Grain Boundary Reflections, Alloy

* Lattice Reflections, Alloy

- Grain Boundary Reflections, Pure

- Lattice Reflections

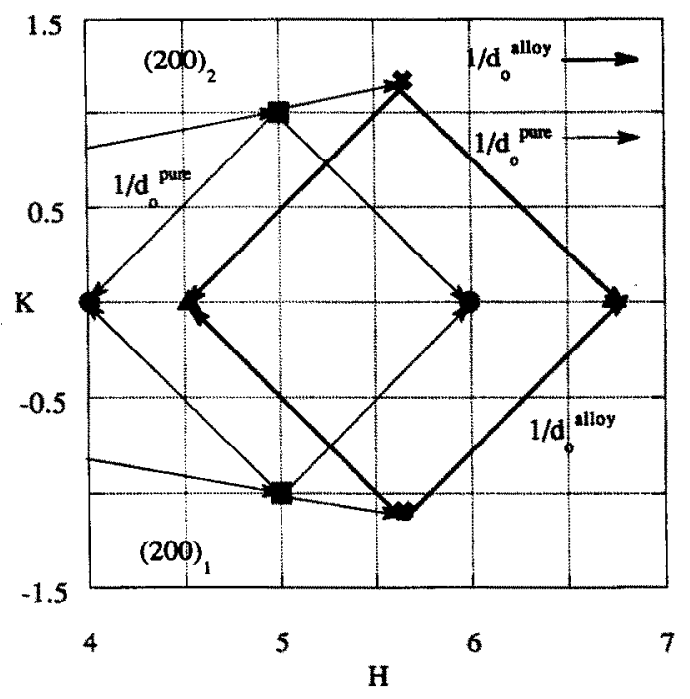

Fig. 2. Expanded (200) region from Fig. 1. Also indicated schematically are the change in peak positions for both lattice and grain boundary reflections, due to alloying. $\mathrm{Xs}$ and solid squares denote the lattice reflections, solid circles and triangles denote grain boundary reflections. A precise location of the positions of the solid triangles, relative to the $\mathrm{Xs}_{\mathrm{s}}$ could provide information on the extent of segregation.

lattice reflection for the alloy. An accurate location of the grain boundary peak position relative to the bulk lattice peak should therefore serve as a check on the compositional homogeneity of the samples and also serve to accurately determine the dislocation spacing. It is interesting to conjecture that one could use the peak position location as a measure of the extent of grain boundary segregation. This will be discussed more extensively in a future publication [13].

Next we note that the distribution of intensities on the BDL is a sensitive function of the detailed atomic structure of the interface. This distribution of intensity is well known and has been extensively measured in a number of systems in the $\Sigma 13$ [001] twist boundary orientation $[2,3,8]$. In particular, for the pure $\mathrm{Au}$ structure this distribution of intensity is known very accurately. In order to determine if the alloying with $\mathrm{Cu}$ produces any significant change in structure of the boundary one needs to compare the intensity distribution in reciprocal space of pure $\mathrm{Au}$ with that of the $\mathrm{Au}-\mathrm{Cu} 10$ at.\% alloy. Figure 3 shows, graphically, the structure factor squared for a number of strong reflections for the $\mathrm{Au}-\mathrm{Cu}$ alloy, the corresponding pure $A u$ values, and the values computed using the free energy minimization (FEM) technique. Clearly, large effects are observed due to the alloying with the $\mathrm{Cu}$. Furthermore it is seen that 


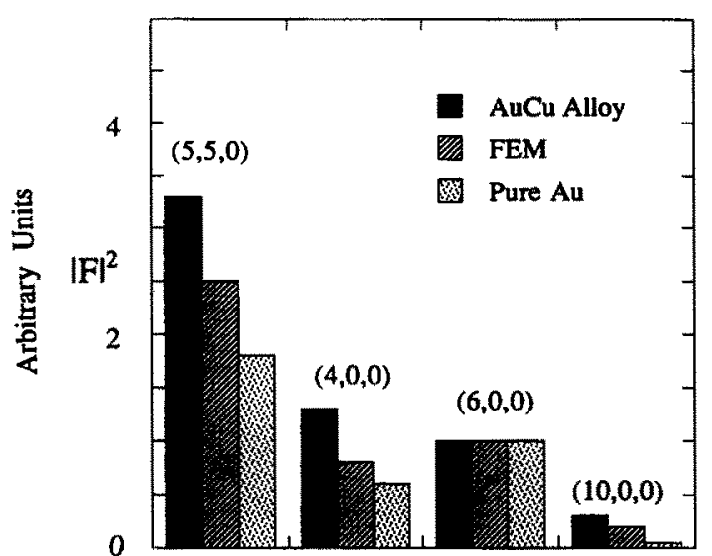

Fig. 3. A comparison of relative structure factors squared [normalized to $(6,0,0)$ reflection] for the alloy, pure Au and calculated (FEM) $\Sigma 13$ [001] twist homophase boundary. Four reflections corresponding to the $\Sigma 13 \mathrm{BDL}$ are shown.

the FEM technique predicts changes whose trends are in agreement with what is observed experimentally, however the precise magnitudes are measured to be significantly larger than those predicted by calculations. It appears that the calculations underestimate the effects of the alloying. Monte Carlo calculations are currently underway in our laboratory [13] to determine whether the differences between experiments and FEM calculations is a shortcoming of the energy minimization technique or has to do with the interaction potentials used in the present simulation.

From a structural point of view the effects due to the alloying are indicated in Fig. 4. The structure shown corresponds to a $\chi^{2}$ value of $\sim 1.5$ with atomic displacements indicated being magnified by a factor of 10. This corresponds to fuller rotational relaxations compared to that of the pure $\mathrm{Au}$ case, therefore implying the existence of somewhat more localized dislocations for the alloyed boundary. The (maximum) magnitude of this additional "displacement" is $\sim 0.06 \AA$. This demonstrates very clearly the power of the X-ray technique and its sensitivity to interfacial atomic arrangements.

The FEM technique also provides site occupation probabilities at the interface for the six symmetrically independent sites in the $\Sigma 13$ boundary however in the present case the site occupation probabilities do not change by more than a factor of about two and this does not give rise to sufficiently large changes in the distribution of measurable intensity in reciprocal space. Hence we are unable, with the present set of measurements, to specify the site occupation probabilities for the six symmetrically independent sites in the $\Sigma 13$ boundary.

\section{Au-PE 26/29}

Little is known about the detailed structure of metal-metal heterophase interfaces. Hwang et al. [14] made observations on the dislocation structure of $\mathrm{Au}-\mathrm{Pd}$ heterophase boundaries as a function of twist angle using TEM. In order to resolve individual dislocations they were necessarily restricted to low angle boundaries. They found, for example, that the dislocation spacing $\left(d_{\mathrm{S}}\right)$ and line orientations $(\sigma)$ for very low angle boundaries obey the relationship first derived by Jesser and Kuhlmann-Wilsdorff [15]:

$$
\begin{gathered}
d_{\mathrm{s}}=\frac{a_{1} a_{2}}{\left[a_{1}^{2}+a_{2}^{2}-2 a_{1} a_{2} \cos (\theta)\right]^{1 / 2}} \\
\sigma=\frac{\pi}{2}-\frac{\theta}{2}-\arctan \frac{\left[a_{1} \sin (\theta)\right]}{a_{2}-a_{1} \cos (\theta)},
\end{gathered}
$$

where $a_{1}$ and $a_{2}$ are the lattice parameters of crystal 1 and 2 , and $\theta$ is the misorientation angle between the two crystals. Here we demonstrate how the thin film X-ray diffraction (TFXD) technique can be applied to extend the study of this problem to higher misorientation angles, and furthermore, upon comparison with atomistic calculations, also provide information on the validity of the calculations in these systems.
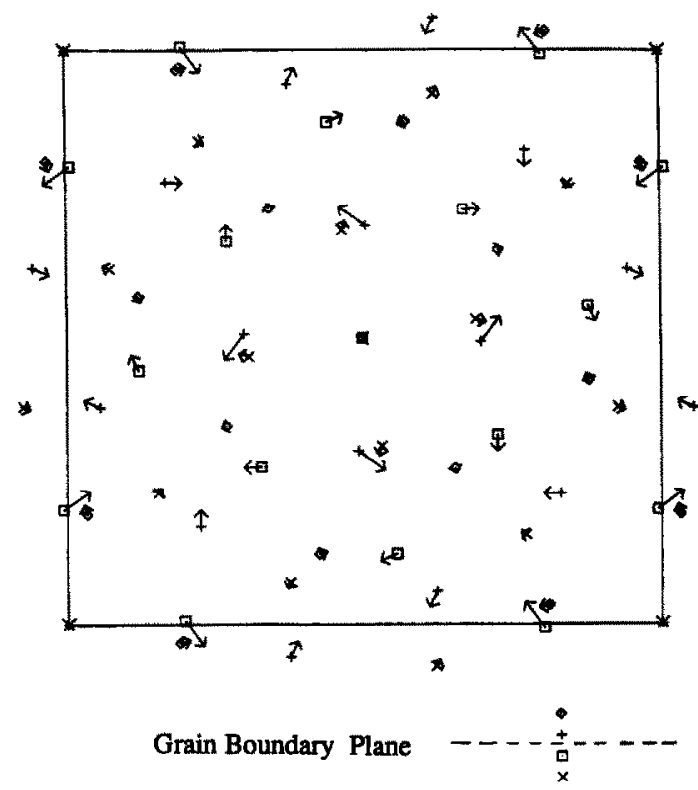

Fig. 4. Atomic structure of the $\Sigma 13$ [001] twist boundary in the $\mathrm{Au}-\mathrm{Cu} 10$ at. \% alloy. Symbols indicate location of relaxed atomic positions in pure $\mathrm{Au}$. Arrow heads indicate the direction of structural relaxations for the alloyed interface, magnified 10 times. The interface is shown as the dotted line between crystals 1 and 2 . 


\section{Sample preparation/X-ray measurements/ calculations}

Our sample preparation technique follows closely that of Refs [7, 13]. Thin film single crystals of $\mathrm{Au}$ (on $\mathrm{Ag}$ ) and $\mathrm{Pd}$ were deposited onto $\mathrm{NaCl}$ substrates in a high vacuum bell jar. Both films were then floated off onto water contained in petri dishes. The $\mathrm{Au} / \mathrm{Ag}$ composite film was first floated onto a flat mica surface, taking care to minimize wrinkles on the gold film. The Pd film was then manipulated on top of this Au film at angles close to the required misorientation. The thin films were then allowed to dry after which they were given a brief anneal at $\sim 300^{\circ} \mathrm{C}$ for a few minutes in a vacuum furnace. This procedure was generally sufficient to form a well defined grain boundary between the two single crystals. Many such boundaries were made and the one with misorientation closest to $10.4^{\circ}(\Sigma 26 / 29)$ and sharpest bulk lattice reflections was chosen for subsequent examination by $\mathrm{X}$-ray diffraction.

The X-ray diffraction measurements were performed using both a rotating anode and the synchrotron source at NSLS/BNLs beamline X20C using $8 \mathrm{keV}$ photons. Further details of the technique are available in Ref. [7].

The calculations were performed using the EAM potentials [6] for $\mathrm{Au}$ and $\mathrm{Pd}$. All calculations were done at $T=0 \mathrm{~K}$ and at constant pressure, corresponding to a minimization of the potential energy of the system. Periodic border conditions were used in the plane of the interface and free Au and Pd surfaces terminated the simulation cell in the direction normal to it. The simulation cell sizes were chosen to correspond to a constrained coincidence site lattice with a maximum strain of $1 \%$, these details will be described in a future publication [13].

\section{Results}

Figure 5 shows the reciprocal lattice (BDL) associated with the $\Sigma 26 / 29 \mathrm{Au}-\mathrm{Pd}$ interface. Measurements were only performed in the $L=0$ plane for a

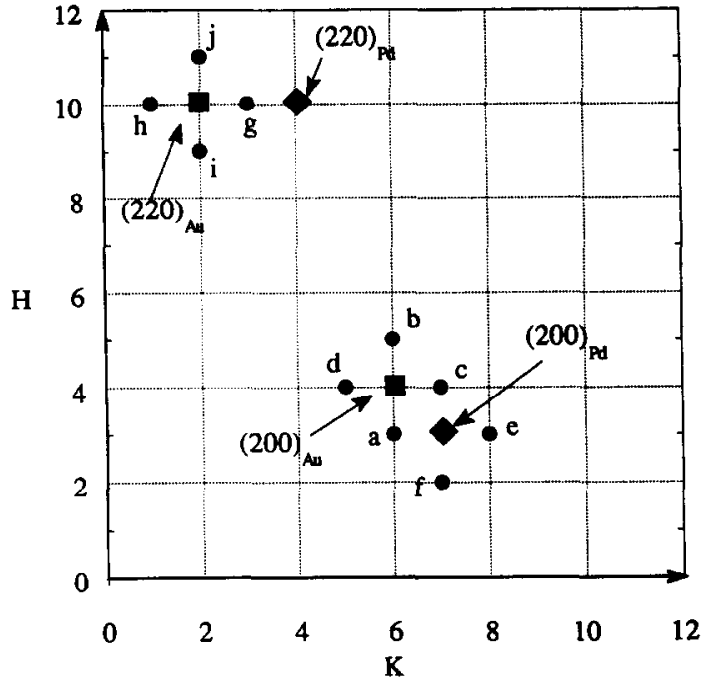

Fig. 5. BDL for $\Sigma 26 / 29 \mathrm{Au}-\mathrm{Pd}$ interphase boundary. Solid squares are the Au lattice reflections, solid diamonds are the Pd lattice reflections. Solid circles denote the interface reflections, these are indexed (a) through (j) and correspond to the reflections indicated in Table 1.

relatively small number of reflections since our purpose here was primarily to check the peak positions (and thereby obtain dislocation spacing and orientation information) and to map out semi-quantitatively the distribution of intensities clustered around the different lattice reflections. Experimental observations were conducted on the reflections indicated by the solid circles, the lattice reflections are indicated by the filled square and diamonds. The magnitudes of the measured and calculated intensities are qualitatively indicated in Table 1 (these are classified as strong- $\mathbf{S}$, medium $-\mathbf{M}$, medium weak-MW and weak-W), and the overall agreement is fair. A similar level of agreement is obtained between the measured and calculated absolute structure factors. Furthermore we observe that all the strong reflections are clustered around lattice reflections, in particular we note that reflections around the Au lattice reflec-

Table 1. Experimentally observed and calculated intensity distribution for the $\Sigma 26 / 29$ $\mathrm{Au}-\mathrm{Pd} 10.4^{\circ}$ interphase boundary. The intensities are classified as strong- $\mathrm{S}$, medium$\mathrm{M}$, medium-weak-MW and weak-W

\begin{tabular}{lccc}
\hline Reflection & Calculations & Measurements & Satellite of lattice reflection \\
\hline (a) $/(6,3)$ & $\mathrm{S}$ & $\mathrm{S}$ & $(200)_{\mathrm{Au}}$ and $(200)_{\mathrm{Pd}}$ \\
$(\mathrm{b}) /(6,5)$ & $\mathrm{S}$ & $\mathrm{S}$ & $(200)_{\mathrm{Au}}$ \\
$(\mathrm{c}) /(7,4)$ & $\mathrm{S}$ & $\mathrm{M}$ & $(200)_{\mathrm{Au}}$ and $(200)_{\mathrm{Pd}}$ \\
$(\mathrm{d}) /(5,4)$ & $\mathrm{S}$ & $\mathrm{M}$ & $(200)_{\mathrm{Au}}$ \\
$(\mathrm{c}) /(8,3)$ & $\mathrm{W}$ & $\mathrm{W}$ & $(200)_{\mathrm{Pd}}$ \\
$(\mathrm{f}) /(7,2)$ & $\mathrm{W}$ & $\mathrm{W}$ & $(200)_{\mathrm{Pd}}$ \\
$(\mathrm{g}) /(3,10)$ & $\mathrm{S}$ & $\mathrm{S}$ & $(200)_{\mathrm{Au}}$ and $(200)_{\mathrm{Pd}}$ \\
$(\mathrm{h}) /(1,10)$ & $\mathrm{S}$ & $\mathrm{MW}$ & $(220)_{\mathrm{Au}}$ \\
$(\mathrm{i}) /(2,9)$ & $\mathrm{W}$ & $\mathrm{W}$ & $(220)_{\mathrm{Au}}$ \\
$(\mathrm{j}) /(4,9)$ & $\mathrm{W}$ & $\mathrm{W}$ & $(220)_{\mathrm{Au}}$ \\
\hline
\end{tabular}


tion are generally stronger than those around the $\mathrm{Pd}$ lattice reflections. Since it is by now well established that the strength of a satellite reflection is a good indication of the magnitudes of the interfacial relaxations in the corresponding lattice [16], it appears that the softer Au lattice exhibits larger relaxation effects than the Pd lattice (Au has a lower shear modulus than Pd). This is further verified by examining the detailed structure of the interface as obtained by molecular statics calculations.

The precise peak positions of the interface satellite reflections are completely consistent with the predictions of the Jesser and Kuhlmann-Wilsdorff formulae (eqn (1)). We note however that the peaks are considerably broader than what is typical in pure homophase systems such as $\mathrm{Au}$ (and is therefore probably due to interdiffusion between the $\mathrm{Au}$ and Pd lattices). Additional calculations at larger misorientation angles are currently underway in our laboratory and preliminary results indicate that the dislocation spacing and line direction change according to eqn (1).

\section{Ag/Cu HETEROPHASE BOUNDARY}

We consider finally the $\mathrm{Ag} / \mathrm{Cu}$ heterophase boundary system. One of the earliest studies in this system was that due to Liu and Balluff [17], who observed secondary grain boundary dislocations for the $\Sigma 4 / 5$ orientation. However detailed experimental information on the primary dislocation structure is completely absent. Gao et al. [18] have studied the $\mathrm{Ag} / \mathrm{Ni}$ [001] twist system exhaustively and find evidence for a cusp (in the energy vs misorientation curve) at the special $\Sigma 4 / 5$ orientation. Detailed quantitative information is however not available using their rotating sphere technique since one can only establish the existence (or absence) of relatively deep minima on this curve. Thus quantitative structural information in this system is still lacking, as is detailed validation of the simulation techniques used. Here we apply the thin film X-ray diffraction technique in conjunction with computer simulation techniques to obtain detailed information on the structure of the interface in this system.

\section{Sample preparation/calculations}

Thin film single crystals were deposited onto $\mathrm{NaCl}$ substrates as described previously. The bicrystals were bonded using the Schober-Balluffi technique [9] in a reducing hydrogen atmosphere as described before, at a temperature of $375^{\circ} \mathrm{C}$ for $15-20 \mathrm{hrs}$. This annealing schedule generally produced well bonded interfaces over a good fraction of the cross-sectional area (as determined by TEM). The bonded thin films were then floated off the $\mathrm{NaCl}$ substrates and mounted onto a thin Kapton film in order to conduct the X-ray diffraction experiments. The details of the procedure will be presented in a future publication.

The calculations were performed using the EAM potentials [6] for $\mathrm{Ag}$ and $\mathrm{Cu}$ and were performed at $T=0 \mathrm{~K}$. The simulation cells chosen were large enough that the strain in the corresponding constrained CSL was generally less than $1 \%$, details on the implication of having a constrained cell on which to perform structure factor calculations will be presented in a future work [13]. Periodic boundary conditions were employed in the plane of the interface and free surfaces terminated the cell in the normal direction. Both low and high angle boundaries were studied by X-ray diffraction and computer simulation, here we focus on the results for the $\Sigma 4 / 5$ orientation.

\section{Results}

Figure 6 shows the reciprocal lattice associated with the $\Sigma 4 / 5$ interface. The radius of the semi-circles is proportional to the intensity measured at the corresponding reciprocal lattice point. Measured values are represented by open semi-circles and calculated values are denoted by solid semi-circles (extremely weak interphase reflections, i.e. no measurable or calculated intensities exist at these BDL

\section{A Lattice Reflections \\ D $\times$ Interface Reflections}

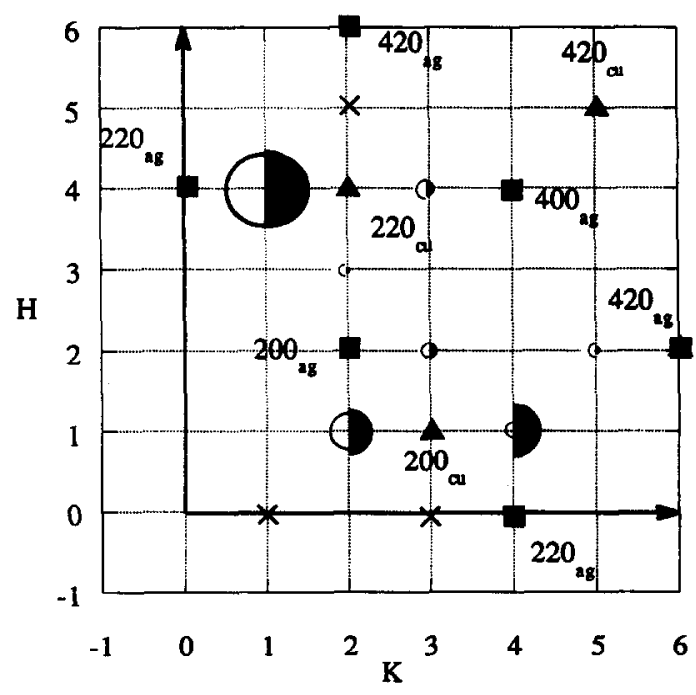

Fig. 6. BDL for $\mathrm{Ag} / \mathrm{Cu} \Sigma 4 / 5$ heterophase boundary. Lattice reflections are indicated by solid triangles $(\mathrm{Cu})$ and squares $(\mathrm{Ag})$. Interface reflections are denoted by open semi-circles (experimental measurements), solid semi-circles (calculations). The diameter of the circles are proportional to the square of the structure factor. Very weak interface reflections are indicated by $\times s$ (implying no observed/calculated intensity exists at these BDL points). 
points, are denoted by large $\times s$ ). We also note that the measurements made correspond to absolute structure factors whose value is a strong indication of the magnitudes of atomic relaxations [16]. It is clear that there is a good overall agreement between the experiments and the calculated structure factors with the exception of reflection $(4,1,0)$. For the $\mathrm{Ag} / \mathrm{Cu} \Sigma 4 / 5$ boundary it is possible to improve the fit to the data and refine the structure by minimizing a test statistic such as $\chi^{2}$. In this model it is assumed that all the relaxation takes place in the $\mathrm{Ag}$ lattice, while the $\mathrm{Cu}$ atoms are assumed to remain in their unrelaxed positions. Upon structural refinement, a $\chi^{2}$ value of $\sim 0.8$ is obtained indicating good agreement between the experiments and the refined structure. Figure 7 shows the results of such a refinement, where we indicate: (a) the unrelaxed $\Sigma 4 / 5$ structure; (b) the structure as predicted by the EAM calculations; and (c) the refined structure obtained from experimental observations. It is seen that the general magnitude and displacement of the atomic relaxations as predicted by the EAM calculations are in fair agreement with those obtained by the experiments. Some slight differences in the structure do however remain.

Some additional comments need to be made with regard to the calculations presented herein. First of all it is clear that some solubility and therefore interdiffusion must exist between the two systems; while this is very small in the bulk at the temperatures of interest it is not clear that this is a negligible effect at the interface, in fact one might argue that whatever differences one observes between the experiments and the calculations (done at $T=0 \mathrm{~K}$ ) could be arising from such higher solubility effects at the interface. We are therefore conducting Monte Carlo calculations for the $\Sigma 4 / 5$ system to provide detailed information on the effects of limited mutual solubility on the structure of the interface. Preliminary Monte Carlo calculations indicate that the calculated overall scale and general direction of relaxations do not change significantly.

Additional calculations at other misorientations indicate that the magnitude of the relaxations are generally small while the directions conform to being of the mixed rotational and dilatational type. This is verified experimentally, for one low angle boundary, where virtually no measurable scattering was detected on the $\mathrm{BDL}$, implying weak atomic relaxations. Hence in the $\mathrm{Ag} / \mathrm{Cu}$ system the structure of the interface corresponds to relaxations that are generally small with erratic dependence on misorientation angle. The only exception to this rule being at the $\Sigma$ $4 / 5$ orientation where large relaxations take place at the interface. This is in striking contrast to the homophase interfaces that have been studied in the past [3], where the structure (magnitude of atomic relaxations and directions) of the boundary changed continuously with misorientation angle starting with large displacements at low angle boundaries, corresponding to well separated, localized dislocations, then decreasing continuously to small displacements at high angle boundaries, with closely spaced, delocalized dislocations. The dependence of structure on misorientation for the two cases discussed above is contrasted in Fig. 8.

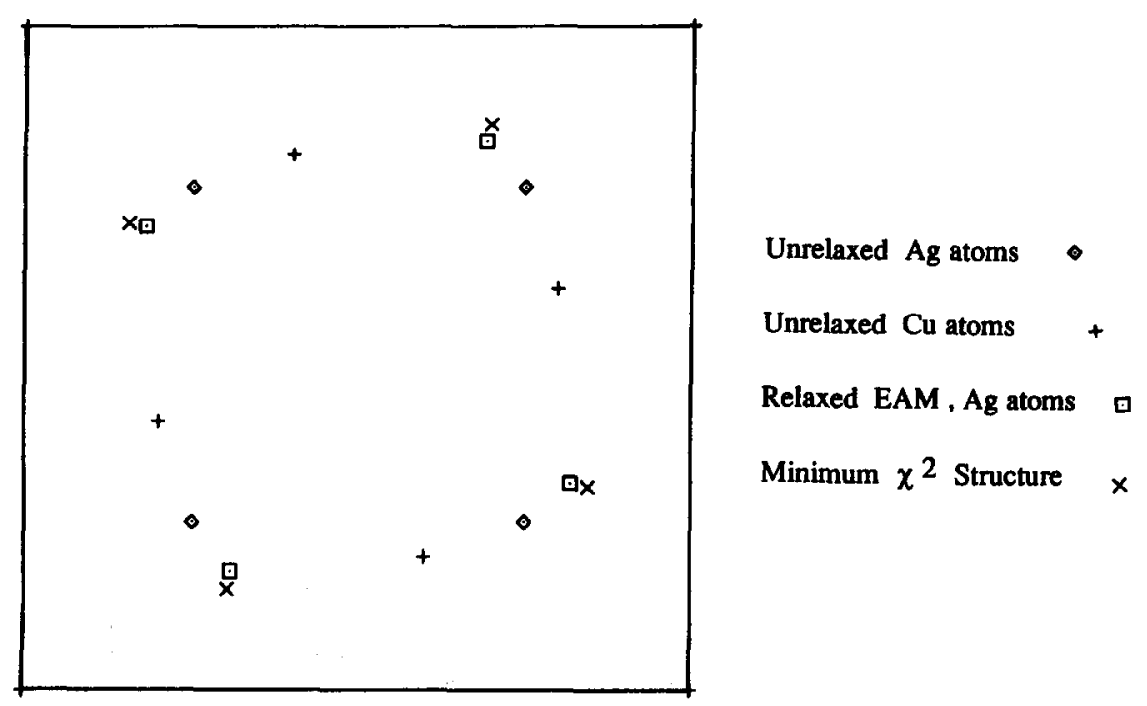

Fig. 7. Unit cell of the $\Sigma 4 / 5$ boundary. Cu atoms denoted by (+) symbols are not allowed to relax. Unrelaxed $\mathrm{Ag}$ atoms are denoted by diamonds, molecular statics relaxed $\mathrm{Ag}$ atoms by squares and minimum $\chi^{2}$ structure $\mathrm{Ag}$ atoms by $\times \mathrm{s}$. 


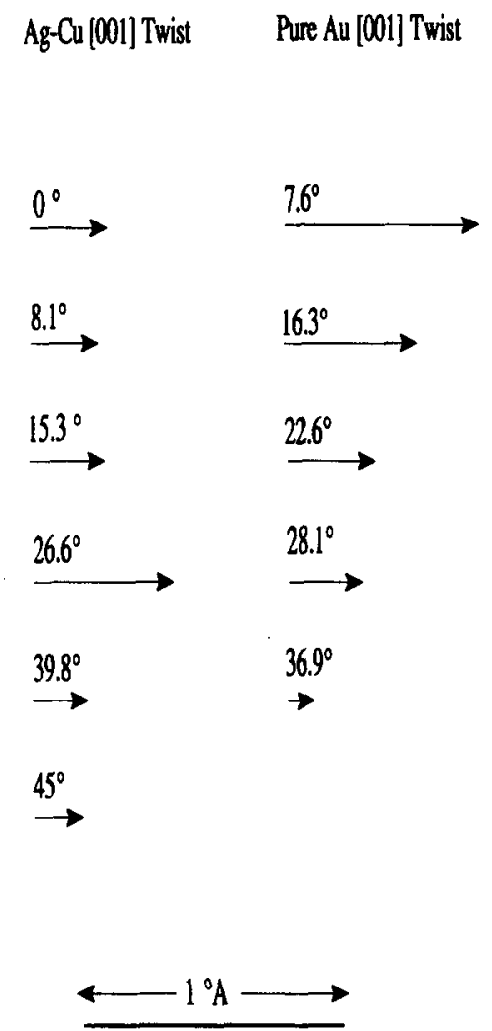

Fig. 8. Maximum planar atomic displacements for pure [001] twist boundaries in pure $\mathrm{Au}$ and in the $\mathrm{Ag} / \mathrm{Cu}$ system. The misorientation angles for the different boundaries are indicated in the figure.

\section{CONCLUSIONS}

Thin film X-ray diffraction in conjunction with computer simulation techniques has been used extensively in the present work to study a variety of metal-metal interfaces to obtain detailed information on their structures. We have demonstrated how the technique may be used to obtain valuable information on the structure of complex interfacial systems.

We have obtained detailed information on the structure of alloyed interfaces, in particular for the $\Sigma$ $13 \mathrm{Au}-\mathrm{Cu}$ system a change in the structure of the interface, relative to that in pure $\mathrm{Au}$, was observed and this was compared to structures obtained by computer simulation techniques. In this system we note that the dominant atomic relaxations correspond to rotational relaxations about O-lattice elements. Furthermore, the magnitudes of the relaxations are slightly larger than those in the pure Au boundary, corresponding to more localized dislocations. Computer simulation techniques predict trends that are in agreement with the experiments, while falling short of agreement with regard to the magnitudes of these effects. In particular we note that slight differences do exist between the results obtained by computer simulation techniques such as FEM and those obtained by experiments. We are presently conducting Monte Carlo calculations to determine the origin of these differences.

For the $\Sigma 26 / 29 \mathrm{Au}-\mathrm{Pd}$ system we have data that, in effect, extends previous TEM work to higher angles indicating the validity of eqns (1) and (2). Experimentally measured structure factors also indicate consistency with computer simulation calcuations. Furthermore, for this boundary, it is found that the softer Au lattice exhibits larger atomic relaxations at the interface than the Pd lattice.

Lastly, for the $\mathrm{Ag} / \mathrm{Cu}$ heterophase boundary, we have provided detailed structural information for the $\Sigma 4 / 5$ orientation both by experiments and calculations. We find that the experimental and calculated structures are quite similar. We further note that the magnitudes of the atomic relaxations for this orientation are quite large, in fact they are even larger than the magnitudes found in corresponding low angle heterophase boundaries (in striking contrast to the pattern of relaxation present in homophase boundaries). This is due to the absence of sufficiently well localized dislocations, at low misorientation angles. In Fig. 8 we contrast the structure of $\mathrm{Ag} / \mathrm{Cu}$ heterophase boundaries with similarly misoriented pure twist boundaries in Au [3]. The maximum planar atomic displacements, at the interface, for different misorientation angles in both of these systems is shown. It is clear that the structure of the interface, as evidenced by the magnitudes of the atomic displacements, changes monotonically for the pure Au twist boundary. For the $\mathrm{Ag} / \mathrm{Cu}$ heterophase boundary, however, it is clear that the atomic relaxations are erratic and generally small, except at the $\Sigma 4 / 5\left(26.6^{\circ}\right)$ orientation.

In the study of very limited solubility heterophase systems we therefore raise the following question. Is the simple dislocation model description of the structure of interfaces, so useful in the study of the pure homophase boundaries, a satisfactory or complete description of the structure of $[001] \mathrm{Ag} / \mathrm{Cu}$ twist boundaries? The experiments described in this work, along with computer simulation studies seem to suggest that it is not.

Acknowledgements-We would like to thank R. W. Balluff for numerous useful discussions on the subject of grain boundary structure. The work was supported by the Department of Energy under Grant Number DE-FG02-87ER45310. Use of the X-ray facilities at NSLS-BNL and CMSE-MIT is gratefully acknowledged. 


\section{REFERENCES}

1. Spence J. C. H., Experimental High-Resolution Electron Microscopy (2nd edn). Oxford University Press, Oxford (1988).

2. Fitzsimmons M. R. and Sass S. L., Acta Metall. 37, 1009 (1989).

3. Majid I., Bristowe P. D. and Balluffi R. W., Phys. Rev. $B$ 40, 2779 (1989)

4. Foiles S. M. and Seidman D. N., in: Materials Interfaces (Edited by D. Wolf and S. Yip). Chapman and Hall, London (1992).

5. Hondros E. D. and Seah M. P., in: Physical Metallurgy (Edited by R. W. Cahn and P. Haasen), pp. 856-931. North-Holland, Amsterdam (1983).

6. Foiles S. M., Baskes M. I. and Daw M. S., Phys. Rev. B 33, 7983 (1986)

7. Majid I. and Bristowe P. D., Phil. Mag. A 66, 73 (1992).

8. Majid I., Counterman C. A., Bristowe P. D. and Balluffi R. W., X-ray diffraction and computer simulation stud- ies of [001] twist boundaries in Au-Ag alloys, accepted for publication in Acta Metall. (1994).

9. Schober T. and Balluffi R. W., Phil. Mag. 20, 511 (1969).

10. Le Sar R., Najafabadi R. and Srolovitz D. J., Phys. Rev. Lett. 63, 624 (1989)

11. Wang H. Y., Najafabadi R. and Srolovitz, D. J., Interface Science 1, 31-47 (1993).

12. Brokman A. and Balluffi R. W., Acta Metall. 31, 1639 (1983).

13. I. Majid et al. (to be published)

14. Hwang M., Laughlin D. E. and Bernstein I. M., Acto Metall. 28, 621 (1980).

15. Jesser W. A. and Kuhlmann-Wilsdorff D., Phys. stat. Solids 21, 533 (1967).

16. Balluffi R. W., Majid I. and Bristowe P. D., Mat. Res. Soc. Proceedings 138, 457 (1989).

17. Liu J. S. and Balluffi R. W., Scripta Metall. 19, 123 (1985).

18. Gao Y., Dregia S. A. and Shewmon P. G., Acta Metall. 37, 1627 (1989). 